\title{
Plasmacytoma cases with unfamiliar clinical features
}

\author{
Ádám J óna ${ }^{1}$, Sándor Barna ${ }^{2}$, Zsófia Miltényi ${ }^{1}$, Lajos Gergely ${ }^{1}$, Árpád I llés ${ }^{1}$, László Váróczy ${ }^{1}$ \\ 1. Division of Hematology, Institute of Internal Medicine, University of Debrecen Medical and Health Science Center, \\ Debrecen, Hungary. 2. Scanomed Ltd., PET-CT Center, University of Debrecen Medical and Health Science Center, \\ Debrecen, Hungary.
}

Correspondence: Ádám Jóna. Address: Division of Hematology, Institute of Internal Medicine, University of Debrecen Medical and Health Science Center, Móricz Zs krt. 98, H-4032 Debrecen, Hungary.E-mail: jona.adam1@gmail.com

Received: September 16, 2012

Accepted: December 4, 2012 Online Published: January 24, 2013

DOI :10.5430/jhm.v3n1p1

URL: http://dx.doi.org/10.5430/jhm.v3n1p1

\begin{abstract}
Plasmacytoma is a relatively rare form of plasma cell dyscrasias with indolent clinical features. Major localizations are in the upper airways and the region of head. Since it represents only a minority of all plasma cell neoplasms, careful histological review should be addressed to the specimens of these patients. In this paper the authors report three cases, developing plasmacytoma with uncommon clinical features.

First patient had an unfamiliar localization of plasmacytoma in the cerebellopontine angle which was first meant to be malignant meningioma and later a large subcutaneous mass was detected in the gluteal region. Second patient developed plasmacytoma after a prostate adenocarcinoma as a secondary malignancy. Third patient, developed plasmacytoma and definite plasma cell myeloma with a coexistence of systemic sclerosis.

Treating patients with chemo-radiotherapy, and irradiating the one with localized disease resulted in complete remission in two out of the three patients. Imaging techniques such as positron emission tomography combined with computed tomography (PET-CT) proved to be useful as tumor exploration tool, however multidisciplinary cooperation is recommended in these cases.
\end{abstract}

\section{Key words}

Plasmacytoma, Plasma cell neoplasm, Central nervous system, Secondary malignancy, Autoimmune disease

\section{Introduction}

Plasma cell neoplasms (PCN) represent a clinically heterogeneous population; they are derived from a malignant proliferation of B-cells, which have undergone somatic hypermutation and immunoglobulin heavy class switching. According to the 2008 World Health Organization (WHO) classification, PCN include monoclonal gammopathy of unknown significance (MGUS); plasma cell myeloma and its variants, which is the most common form; plasmacytoma; immunoglobulin deposition diseases and osteosclerotic myeloma ${ }^{[1]}$. Plasmacytoma is further divided into solitary plasmacytoma of the bone and extraosseous (extramedullary) plasmacytoma. Extraosseous plasmacytoma represents only $4 \%$ of all PCN ${ }^{[2]}$. Generally it is a solid tumor, no bone marrow involvement and systemic development can be seen. While solitary plasmacytoma of the bone arises from bone marrow, extraosseous plasmacytoma derives from the submucosal layer of tissues. Most frequent localizations are: upper airways, gastrointestinal tract, urinary tract, lungs, lymph nodes and $\operatorname{skin}^{[3]}$. It can progress to plasma cell myeloma with a frequency of $15-30 \%$, retrospectively ${ }^{[4-5]}$. The diagnosis is usually based on histopathological examination after a fine needle aspiration or biopsy. The tumor is 
characterized by mature CD138+ plasma cells with slight pleomorphism and low mitotic index ${ }^{[6]}$. The disease is clinically indolent, responses well to radiotherapy, external beam radiotherapy gives good disease control, however it is disposed for recidive. Differential diagnosis may include Castleman disease, mucosa associated lymphoid tissue (MALT) lymphoma, and extramedullary involvement of other PCNs. As these tumors are rare and can be misdiagnosed with other types of non-Hodgkin's lymphoma, a histological review is strongly recommended by a histopathologist with a special interest in lymphoproliferative disorders.

\section{Case presentations}

\section{Case 1}

A 65-year-old male patient underwent neurosurgery due to a tumor, localized in the cerebellopontine angle in 1998. Histological examination revealed malignant meningioma, therefore he received postoperative irradiation. In December, 2007, he presented again with dizziness and ataxia. Cranial magnetic resonance imaging (MRI) proved a local recidive (see Figure 1). As a result of the biopsy from another neurosurgery, he had plasmacytoma (see Figure 2); therefore his former biopsy was revised, which proved to be plasmacytoma also. Further staging examinations were begun in January 2008. There were no elevated number of plasma cells present in the bone marrow and no monoclonal protein was found in his serum and urine samples. However, a large subcutaneous mass was detected in his right gluteal region, which was found to be plasmacytoma on the cytological examination again. Afterwards, he received three cycles of Thalidomide-Dexamethasone + intrathecal Dexamethasone treatment between February and April of 2008, which was followed by the local irradiation of the gluteal mass in May 2008. As the result of these treatments, a complete remission was achieved and no relapse has been detected so far.

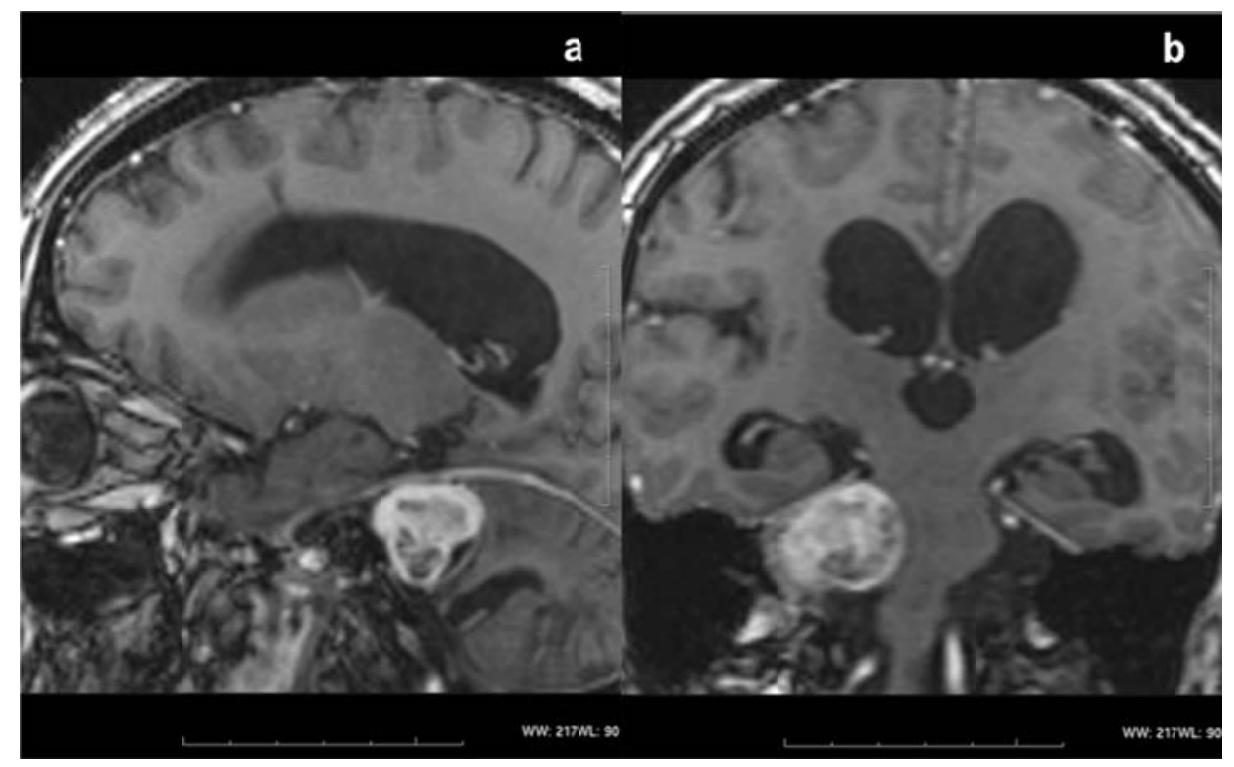

Figure 1.(a) Saggital and (b) cranial magnetic resonance imaging (MRI) scan of plasmacytoma, localized in the cerebellopontine angle

\section{Case 2}

A 57-year-old male patient had diabetes mellitus and stroke in his former history. In August, 2007, he underwent radical prostatectomy due to adenocarcinoma. Afterwards, positron emission tomography combined with computed tomography (PET-CT) scan detected a pathological lymph node in his right axilla (see Figure 3), therefore he underwent 
lymphadenectomy. The histology confirmed plasmacytoma (see Figure 4). Hematological examination was begun in January, 2008. His bone marrow biopsy result was negative, he had no paraprotein and no lytic bone process could have been observed. He received irradiation with a total dose of $40 \mathrm{~Gy}$ to his right axilla between February and March of 2008. Since April, 2008 no relapse have been observed.

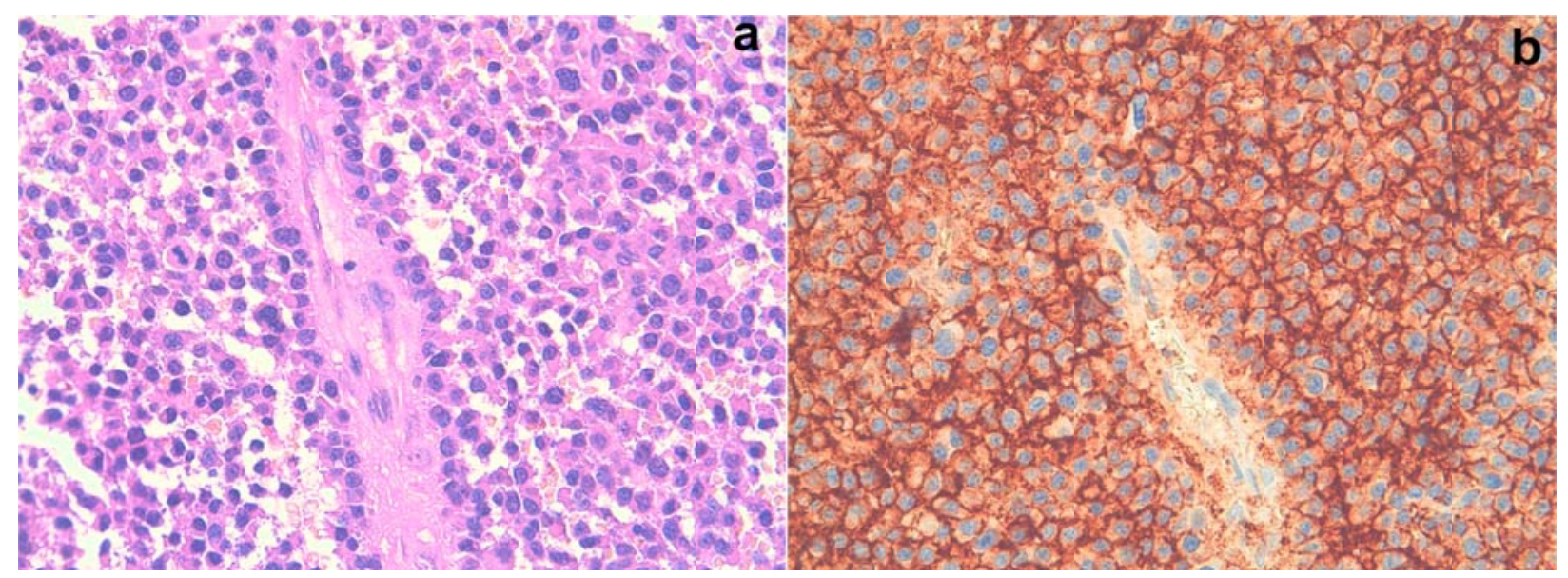

Figure 2. Histological image of plasmacytoma in the cerebellopontine angle (hematoxyline-eosine, (40x magnification) (a) and CD138+, (60x magnification) (b) staining)

Figure 3. PET-CT scan of plasmacytoma localized in the right axilla (coronal section)

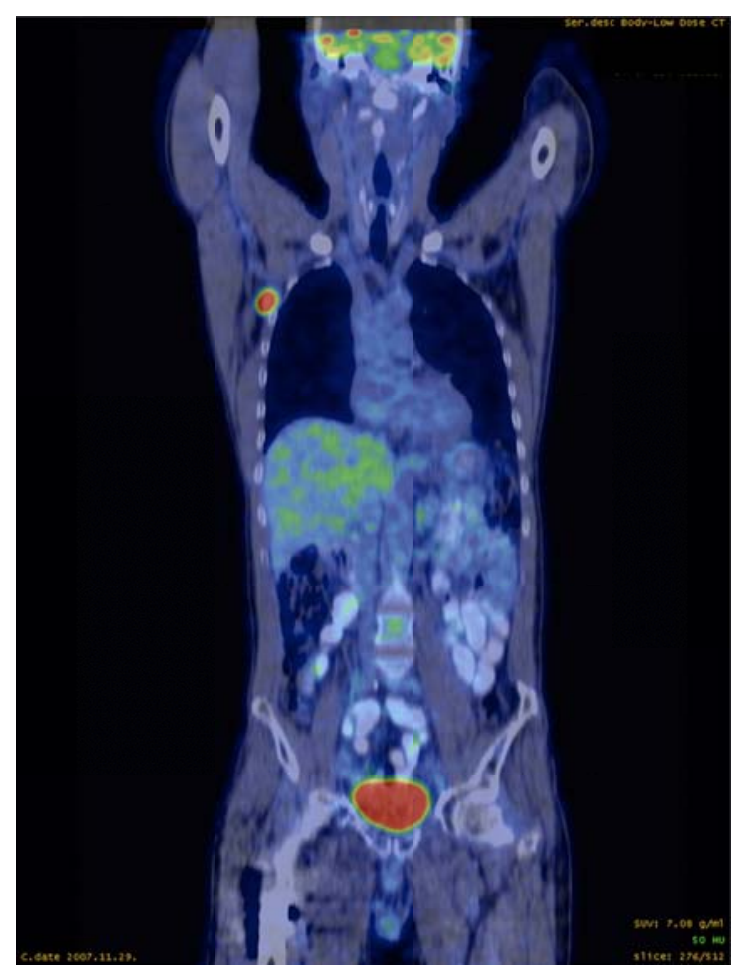




\section{Case 3}

A 36-year-old female patient, born in 1974, was diagnosed with diffuse goiter and sarcoidosis in 1996 . She had an intrauterin fetal death through a 7 months' long pregnancy in 1997. She was later diagnosed with limited form of systemic sclerosis and antiphospholipid syndrome in 1998, with pulmonary fibrosis and fibrotising alveolitis in 1999, which she received cyclophosphamide treatment for. In 2006, she underwent caesarean section due to toxemia, her newborn weighed 400g. Due to progression of scleroderma symptoms and alveolitis she received azathioprin in 2006. Afterwards she underwent a neurosurgery in March 2008, due to a tumor destroying her third cervical vertebra. The histological examination confirmed plasmacytoma again. She was seen by a hematologist in April, 2008 at first. There was no significant elevation of the number of plasma cells in the bone marrow, however, IgG kappa paraprotein was found in the sera with a concentration of $30 \mathrm{~g} / \mathrm{L}$. This patient fulfilled the criteria of plasma cell myeloma, as paraprotein was present also with plasmacytoma, however bone marrow was not involved. Her disease was Salmon-Durie stage IIA. International Staging System (ISS) stage I (ß2 microglobulin $1.6 \mathrm{mg} / \mathrm{L}$, albumin $38 \mathrm{~g} / \mathrm{L}$ ). PET-CT scan showed a pathological mass in L2 vertebra and in the left pulmonary apex (see Figure 5). She received irradiation therapy with a total dose of $38 \mathrm{~Gy}$ to the C3 vertebra. From June, 2008 she received two cycles of Thalidomide-Dexamethasone protocol, but her therapeutic response was not satisfying (IgG kappa: 18 g/L). Between September, 2008 and April, 2009 she received 4 cycles of Bortezomib-Dexamethasone and 4 cycles of Bortezomib-Dexamethasone-Cyclophosphamid treatment. She could achieve a good partial remission (IgG kappa: $9.67 \mathrm{~g} / \mathrm{L}$ ), but then she progressed again within a few weeks (IgG kappa: $31.7 \mathrm{~g} / \mathrm{L}$ ). She was referred for autologous stem cell transplantation. Unfortunately, she developed fatal pneumonia and septic shock in August, 2009.

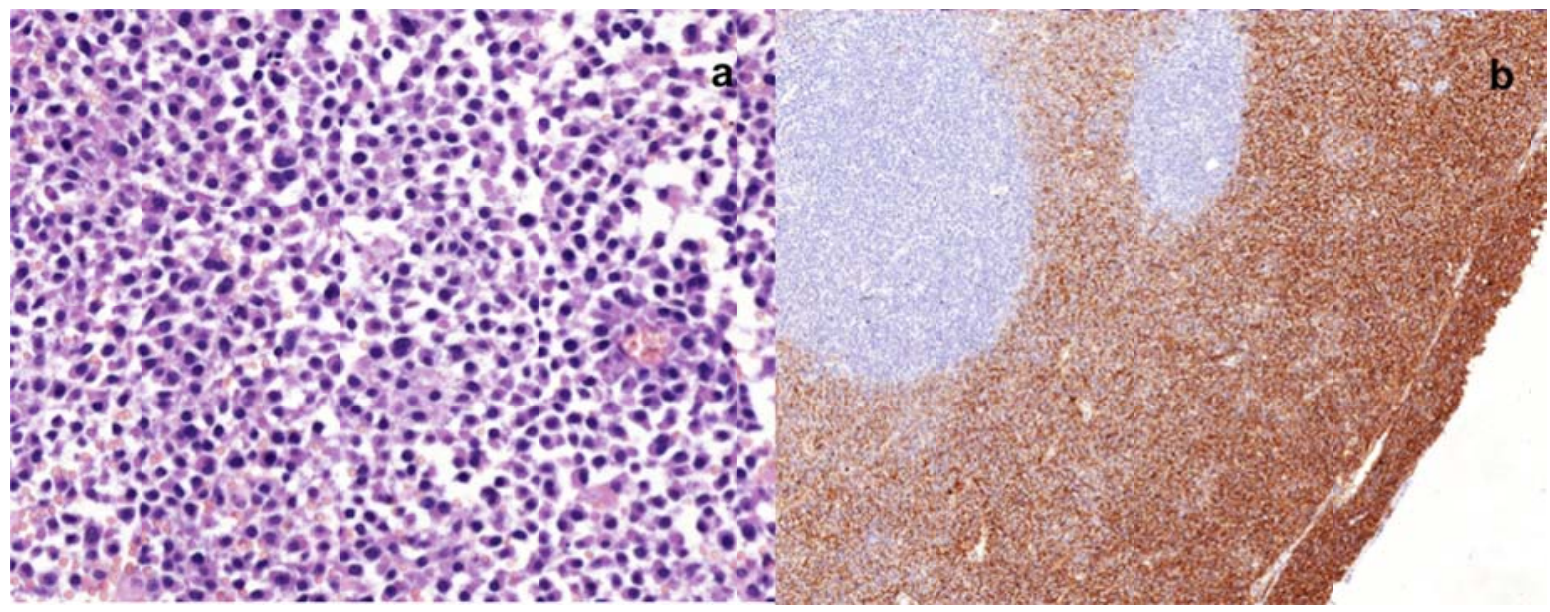

Figure 4. Histological image of plasmacytoma in the right axilla (hematoxyline-eosine(a) and CD138+ (b) staining, 100x magnification)

\section{Discussion}

Plasmacytoma is a rare plasma cell neoplasm with indolent clinical features. In three uncommon cases significant diagnostic and therapeutic efforts have been evolved.

Number of reported cases of plasmacytoma in the central nervous system (CNS) is limited ${ }^{[7,8]}$. Wellwood et al. described a case of plasma cell leukemia relapsing as plasmacytoma in the testis and meninges. Since, no CNS prophylaxis was used during initial treatment; they figured CNS was a sanctuary site for the disease during initial therapy. Bruns et al. reported an uncommon bifocal localization of plasmacytoma. First, the tumor of the knee was misdiagnosed but later, due to diagnosing the CNS involvement, the first specimen was revised. As being a localized tumor, the patient received radiation 
therapy. Similarly, our patient's CNS involvement was first misdiagnosed. Based on his late local recidive followed by a subcutaneous gluteal involvement, this patient's disease can be concerned as plasmacytoma in two localizations. This case brings one's attention to revise previous specimens, when recognizing a rare tumor at an uncommon site.

Figure 5. PET-CT scan of pathological mass in $\mathrm{L} 2$ vertebra and in the left pulmonary apex

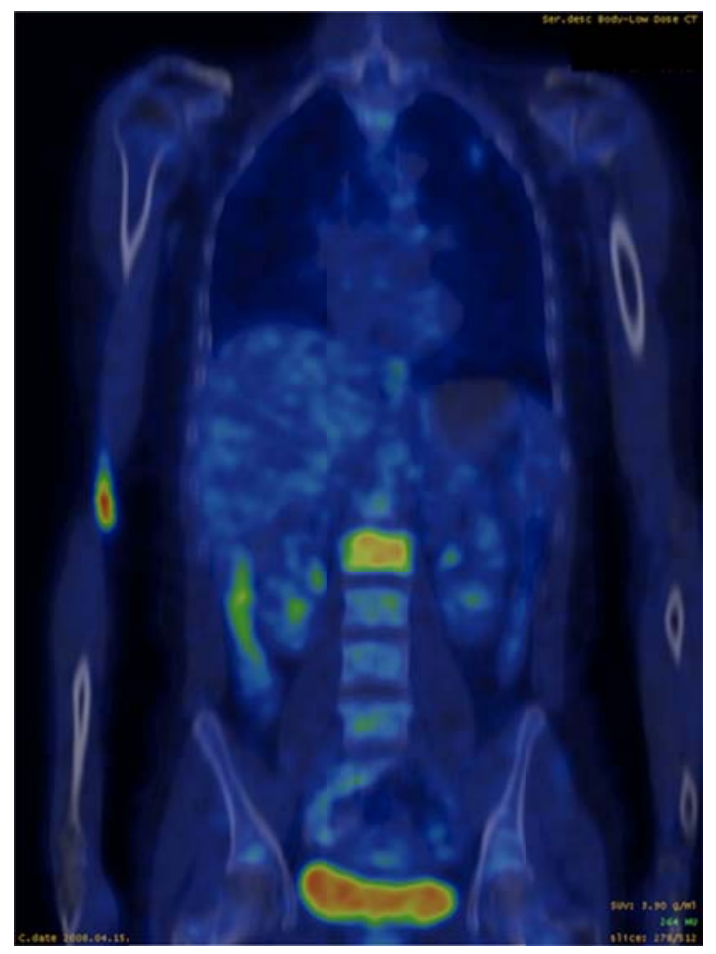

Prostate carcinoma is usually treated either by surgery or irradiation, which is associated a significant but still small risk enhancement of secondary solid tumors ${ }^{[9]}$. A recent paper compared the risk of secondary malignancies of prostate cancer after irradiation therapy vs. prostatectomy. Irradiation induced more secondary malignancies but significantly only after 5 and 10 years. The most common affected sites were bladder, lymphoproliferative, and sarcoma in both groups ${ }^{[10]}$. Plasmacytomas are usually secondary tumor of plasma cell myeloma. Therefore, we think the patient developing plasmacytoma after prostate adenocarcinoma can be concerned as having two independent tumors;however this patient must have an increased risk of developing malignancies.

The coexistence of autoimmune diseases and hematological malignancies is often reported in the literature. Our workgroup published some data previously on the increased risk of autoimmune diseases in patients with malignant lymphomas ${ }^{[11]}$. Of non-Hodgkin lymphoma patients $12.9 \%$ developed autoimmune disorders, while of Hodgkin patients $11.5 \%$ had associated autoimmune disease compared to 5-6\% prevalence in the general population. Sjögren syndrome was found to be the most frequent disorder. However, the connection between plasma cell disorders and autoimmune diseases are reported much less frequently ${ }^{[12]}$. A publication shows some connection between Sjögren syndrome and systemic lupus erythematosus (SLE), in which serum monoclonal immunglobulins have been shown ${ }^{[13]}$.The coexistence of SLE and plasma cell myeloma have been also reported sporadically ${ }^{[14]}$.

The connection between autoimmune disorders and malignant lymphoma may be related to impaired $\mathrm{B}$ and $\mathrm{T}$ cell function, while coexistence of systemic sclerosis and myeloma may be related to an unknown genetic lesion.

Treating and maintaining our patients required careful handling and multidisciplinary cooperation. Two out of three patients received combined chemo-radiotherapy, one patient had localized disease, which she received only irradiation for. 
As of today two patients are in complete remission, one patient died in sepsis. As PET-CT was used as a tumor exploration tool it was found to be useful to detect plasmacytoma in the latter two cases.

\section{Source(s) of support}

This work was supported by Hungarian Ministry of Health (ETT) Grant 200/2009.

\section{Conflict of interest}

The authors declare that they have no conflict of interest.

\section{References}

[1] Swerdlow S H, Campo E, Harris N L, et al. WHO Classification of Tumours of Haematopoietic and Lymphoid Tissues. 4th ed. Lyon, France: International Agency for Research on Cancer; 2008.

[2] Reed V,Shah J, Medeiros L, et al. Solitary plasmacytomas: Outcome and prognostic factors after definitive radiation therapy. Cancer. 2011. http://dx.doi.org/10.1002/cncr.26031

[3] Dores G M, Landgren O, McGlynn K A, et al. Plasmacytoma of bone, extramedullary plasmacytoma, and multiple myeloma: incidence and survival in the United States, 1992-2004. Br J Haematol. 2009; 144(1):86-94.

PMid:19016727 http://dx.doi.org/10.1111/j.1365-2141.2008.07421.x

[4] Soutar R, Lucraft H, Jackson G, et al. Guidelines on the diagnosis and management of solitary plasmacytoma of bone and solitary extramedullary plasmacytoma. Br J Haematol. 2004; 124(6): 717-726. PMid:15009059 http://dx.doi.org/10.1111/j.1365-2141.2004.04834.x

[5] Lorsbach R B, Hsi E D, Dogan A, Fend F. Plasma cell myeloma and related neoplasms. Am J Clin Pathol. 2011 ; 136: $186-182$. PMid:21757591 http://dx.doi.org/10.1309/AJCPENJ68FFBRIYB

[6] Kumar P V, Owji S M, Talei A R. Malekhusseini S, Extramedullary plasmacytoma. Fine needle aspiration findings. Acta Cytol. 1997; 41(2): 364-368.

PMid:9100768 http://dx.doi.org/10.1159/000332526

[7] Bruns F, Janssen S, Laenger F, Dobbelstein C, Meyer A. Extramedullary Plasmocytoma: A Rare Case with Bifocal Manifestation at Uncommon Sites. Anticancer Res. 2010; 30(5):1779-1781.PMid:20592378

[8] Wellwood J, Taylor K, Wright S, et al. Relapsed plasma cell leukaemia presenting with aggressive extramedullary plasmacytomas in the meninges and testis--a missed opportunity for induction CNS prophylaxis. Leuk Lymphoma. 2002; 43(4): 893-896. PMid:12153182 http://dx.doi.org/10.1080/10428190290017060

[9] Brenner D J, Curtis R E, Hall E J, et al. Second Malignancies in Prostate Carcinoma Patientsafter Radiotherapy Compared with Surgery. Cancer. 2000; 88(2):396-406. http://dx.doi.org/10.1002/(SICI)1097-0142(20000115)88:2<398::AID-CNCR22>3.0.CO;2-V

[10] Huang J, Kestin L L, Ye H, et al. Analysis of second malignancies after modern radiotherapy versus prostatectomy for localized prostate cancer. Radiother Oncol. 2011; 98(1):81-86. PMid:20951450 http://dx.doi.org/10.1016/j.radonc.2010.09.012

[11] Varoczy L, Payer E, Kadar Z, et al. Malignant lymphomas and autoimmunity-a single center experience from Hungary. Clin Rheumatol. 2012; 31(2):219-224. Epub 2011 Jul 2017.

[12] Urbanska-Rys H, Robak E, Kordek R, et al. Multiple myeloma in a patient with systemic lupus erythematosus, myasthenia gravis and non-familial diffuse palmoplantar keratoderma. Leuk Lymphoma. 2004; 45(9): 1913-1918.

PMid:15223654 http://dx.doi.org/10.1080/10428190410001663581

[13] Youinou P, Le C R, Dueymes M. Autoimmune diseases and monoclonal gammopathies. Clin Exp Rheumatol.1996; 14: S55-58. PMid:8722201

[14] Afeltra A, Amoroso A, Garzia P, Systemic lupus erythematosus and multiple myeloma: a rare association. Semin Arthritis Rheum. 1997; 26(6):845-849. http://dx.doi.org/10.1016/S0049-0172(97)80029-7 